\section{Evaluation of Cell Membrane Electro- permeabilization by Means of a Nonpermeant Cytotoxic Agent}

BioTechniques 28:921-926 (May 2000)

\begin{abstract}
For the evaluation of cell membrane electropermeabilization, cells are usually exposed to electric pulses in the presence of propidium iodide, a fluorescent dye activated by binding to cellular DNA. The fraction of permeabilized cells is then determined using a flow cytometer. This widely established method has several drawbacks: (i) an arbitrary choice of minimum fluorescence intensity for characterization of permeabilized cells; (ii) the inability to detect cells disintegrated because of intense electropermeabilization; and (iii) false detection of cellular ghosts devoid of fluorescence because of leakage of DNA caused by electropermeabilization. Here, we present a simple and inexpensive method that eliminates these drawbacks. The method is based on the use of a cytotoxic agent that cannot permeate through an intact plasma membrane and thus leads to selective death of the electropermeabilized cells. The amount of nonpermeabilized cells is then determined by a suitable viability test. Bleomycin at a 5-nM concentration causes no statistically significant effect on cell survival in the absence of electric pulses, yet this concentration is sufficient for lethal toxicity in electropermeabilized cells. The amount of cells surviving the exposure relative to the control gives a reliable value of the fraction of nonpermeabilized cells.
\end{abstract}

\section{INTRODUCTION}

Exposing cells to electric pulses can render their plasma membranes permeable to otherwise nonpermeant molecules. This phenomenon, referred to as membrane electropermeabilization (sometimes called electroporation), depends on the number of pulses delivered, pulse duration and pulse ampli- tude. With these parameters properly chosen, electropermeabilization is reversible, and the cells return to their normal physiological state. Internalization of various molecules using this method has found applications in oncology (9), genetics (11), immunology (7) and cell biology (4).

Before the method is routinely used, pulse parameters for the best possible yield of reversible permeabilization have to be determined. In general, with a given number and duration of pulses, pulse amplitudes that are too low do not lead to permeabilization at all, while amplitudes that are too high lead mostly to irreversible permeabilization and therefore to cell death. Quantitatively, this can be characterized by two pulse amplitudes, as shown in Figure 1 - the first leading to permeabilization of $50 \%$ of the cell population $\left(\mathrm{P}_{50 \%}\right)$ and the second leading to death of $50 \%$ of the cell population $\left(\mathrm{D}_{50 \%}\right)$.

$\mathrm{D}_{50 \%}$ is determined by the viability of the cells exposed to electric pulses; this is easily assessed either by their cloning efficiency (13) or by an MTT assay (5). $\mathrm{P}_{50 \%}$ is usually evaluated through the uptake of propidium iodide, a fluorescent dye activated by binding to DNA (2). Other dyes sometimes used for this purpose include trypan blue (14) and lucifer yellow (6). The latter two methods, however, have considerable weaknesses. Namely, trypan blue is a stain actively excluded from living cells, and the process of exclusion depends on the level of cellular ATP. Since the latter is perturbed by electropermeabilization, this biases a quantitative evaluation. Lucifer yellow fluoresces both inside and outside the cell, and thus the cell suspension has to be washed and centrifuged two or three consecutive times before the measurement. Because the centrifugations are performed immediately after electropermeabilization, this might lead to the death of certain cells that would otherwise survive the treatment.

Because propidium iodide binds to cellular DNA, it remains in the cell once internalized. In addition, propidium iodide only fluoresces when internalized, and thus no washing of the cell suspension after the exposure to electric pulses is required. The percentage of permeabilized cells is then efficiently mea- sured on a flow cytometer (12). Because of this, the propidium iodide method is widely established today. However, despite its advantages over the alternative methods, it has three substantial drawbacks (Figure 2): (i) though various calibrations can be performed, the minimum fluorescence intensity for characterization of electropermeabilized cells is largely chosen arbitrarily; (ii) the cells that disintegrated because of intense electropermeabilization cannot be detected, which biases the calculated percentage of electropermeabilized cells; and (iii) the cellular ghosts that are devoid of fluorescence because of leakage of DNA caused by electropermeabilization are incorrectly detected as nonpermeabilized cells.

Here, we present a simple and inexpensive method that eliminates the drawbacks of the propidium iodide method. Exposure to electric pulses in the presence of bleomycin, a cytotoxic agent that cannot permeate through an intact plasma membrane (10), leads to selective death of the electropermeabilized cells, and the survival is determined by the cloning efficiency. In the absence of electric pulses, a 5-nM concentration of bleomycin causes no statistically significant effect on cell survival, yet this concentration is sufficient for lethal toxicity in electropermeabilized cells. The cloning efficiency normalized to the control gives a reliable value of the fraction of nonpermeabi-

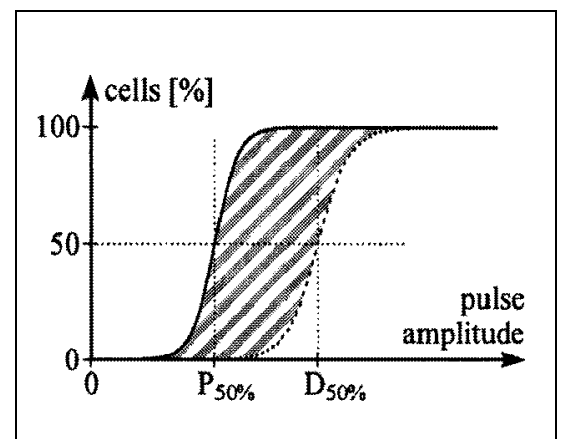

Figure 1. Electropermeabilization (solid) and cell death (dashed) as functions of electric pulse amplitude (a hypothetical situation). Pulse amplitude is best evaluated as the voltageto-distance ratio. We denote the pulse amplitudes corresponding to $50 \%$ values on the two curves as the $\mathrm{P}_{50 \%}$ and $\mathrm{D}_{50 \%}$. If permeabilization is assumed to be the only reason for cell death, reversibly permeabilized cells occupy the entire area between the two curves (hatched). 


\section{Short Technical Reports}

lized cells. By subtracting this value from $100 \%$ at each pulse amplitude, one obtains the cell permeabilization as a function of the pulse amplitude. As with the propidium iodide method, $\mathrm{P}_{50 \%}$ can then be assessed by nonlinear regression of the experimental data.

\section{MATERIALS AND METHODS}

\section{Cells}

DC3F cells, a line of spontaneously transformed Chinese hamster fibroblasts (3), were grown in monolayers at $37^{\circ} \mathrm{C}$ and $5 \% \mathrm{CO}_{2}$ in a Universal Jacketed Incubator (Forma Scientific, Marietta, OH, USA). Flasks $\left(150 \mathrm{~cm}^{2}\right)$ were used for general cultivation, and 60$\mathrm{mm}$ petri dishes were used for cloning efficiency assays (both from TPP, Trasadingen, Switzerland). The culture medium consisted of Eagle minimum essential medium (EMEM) 41090 supplemented with $10 \%$ fetal bovine serum (both from Life Technologies, Rockville, MD, USA), $100 \mathrm{U} / \mathrm{mL}$ penicillin and $125 \mu \mathrm{g} / \mathrm{mL}$ streptomycin (both from Sarbach/Solvay Pharma, Brussels, Belgium).

\section{Exposure to Electric Pulses}

After trypsination with trypsinEDTA (Life Technologies), cells were centrifuged for $5 \mathrm{~min}$ at $1000 \mathrm{rpm}$ in a C312 centrifuge (Jouan, Saint Herblain, France) and resuspended at 2 $\times 10^{7}$ cells/mL in Spinner minimum essential medium (SMEM) 21385; (Life Technologies), which is a calcium-depleted modification of EMEM. A 50$\mu \mathrm{L}$ droplet of the cell suspension was placed between two flat stainless steel electrodes $2 \mathrm{~mm}$ apart, and monophasic rectangular electric pulses were then applied with a GHT 1287B electropulsator (Jouan).

\section{Determination of Electropermeabi- lization with Propidium Iodide}

Before the exposure to electric pulses, propidium iodide was added to the suspension in the amount leading to $100-\mu \mathrm{M}$ propidium iodide concentration. After the exposure, cells were incubated for $10 \mathrm{~min}$ and resuspended in
PBS (Life Technologies). A FACSort ${ }^{\mathrm{TM}}$ flow cytometer (Becton Dickinson, Franklin Lakes, NJ, USA) was used to assess the percentage of permeabilized cells (Figure 2). Excitation was set at 488-nm wavelength and emission was detected at $640 \mathrm{~nm}$.

\section{Determination of Electropermeabi- lization with Bleomycin}

Before the exposure to electric pulses, bleomycin (Laboratoires Roger Bellon, France) was added to the suspension in the amount leading to a 5-nM bleomycin concentration. After the exposure, cells were incubated for $10 \mathrm{~min}$ at room temperature and then resuspended in SMEM. After an additional $30 \mathrm{~min}$, cells were diluted in the culture medium to 100 cells $/ \mathrm{mL}$, and $4 \mathrm{~mL}$ suspension were transferred into each 60$\mathrm{mm}$ petri dish where the cells were grown for five days. Cells were then fixed by a 15-min exposure to $100 \%$ ethanol (Carlo Erba Reagenti, Milan, Italy) and stained for 15 min with $1 \%$ crystal violet (Sigma). Clone colonies were counted and normalized to the

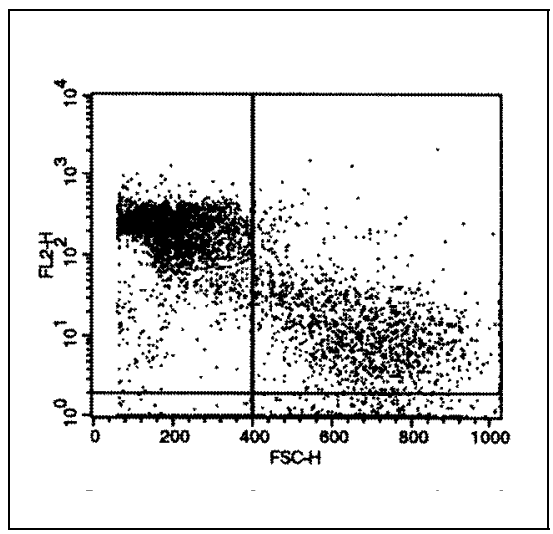

Figure 2. An example of a flow cytometer plot in the evaluation of electropermeabilization with the propidium iodide method. The $\mathrm{x}$-axis gives the forward scattering (reflecting the size) of the analyzed particle (FSC-H), and the y-axis gives its fluorescence (FL2-H); both are in arbitrary units. Above the horizontal limiter, the particles have sufficient fluorescence to qualify as permeabilized, while to the right of the vertical limiter, the particles are large enough to qualify as cells. Debris of the cells disintegrated by intense permeabilization is mostly found in the thick cloud in the top left quadrant, and the ghosts devoid of DNA are in the bottom right quadrant. The measurement shown above was obtained with one monophasic rectangular pulse of 1-ms duration and $1200 \mathrm{~V} / \mathrm{cm}$ amplitude. control (no pulses) to get the percentage of cells surviving the exposure to electric pulses in suspension with $5 \mathrm{nM}$ bleomycin. By subtracting this percentage from $100 \%$, the percentage of permeabilized cells was obtained.

\section{Treatment of Experimental Data}

All experiments were repeated three times at intervals of several days or more. For each experimental point, mean and standard deviation (N-1) were determined. Using nonlinear regression, the data obtained by the propidium iodide method were fitted to a four-parameter sigmoid curve

$$
\text { Eq. } 1 y(x)=y_{\mathrm{MIN}}+\frac{y_{\mathrm{MAX}}-y_{\mathrm{MIN}}}{1+\exp \left[\left(x_{\mathrm{c}}-x\right) / b\right]}
$$

where $x$ is the pulse amplitude, $y$ is the percentage of permeabilized cells, while $x_{\mathrm{c}}, y_{\mathrm{MIN}}, y_{\mathrm{MAX}}$ and $b$ are the four parameters determining the shape of the sigmoid curve. Similarly, the data obtained by the bleomycin method were fitted to a two-parameter sigmoid curve

$$
\text { Eq. } 2 y(x)=y_{\mathrm{MIN}}+\frac{100 \%}{1+\exp \left[\left(x_{\mathrm{c}}-x\right) / b\right]}
$$

with $x$ and $y$ as above, and $x_{\mathrm{c}}=$ $\mathrm{P}_{50 \%}$ (bleomycin) and $b$ the two parameters of the sigmoid curve. The use of a two-parameter sigmoid curve is justified by the fact that the bleomycin curves always span from 0\%-100\%. Furthermore, the parameters obtained by fitting the same data to both sigmoid curves differ only insignificantly (e.g., the values of $\mathrm{P}_{50 \%}$ differ by less than one part in a hundred).

\section{RESULTS}

Cell membrane electropermeabilization as assessed by the propidium iodide method and by the bleomycin method was determined for monophasic rectangular pulses with four typical sets of parameters: one $100-\mu$ s pulse, one 1-ms pulse, eight $100-\mu$ s pulses with $1-\mathrm{Hz}$ repetition frequency and eight $1-\mathrm{ms}$ pulses with $1-\mathrm{Hz}$ repetition frequency. Figure 3 shows the results of these measurements. 


\section{Short Technical Reports}

\section{DISCUSSION}

The bleomycin method introduced in this paper has several advantages compared with the established propidium iodide method. The first of these advantages is shown in Figure 3, where the bleomycin ("BLM") curve spans from $0 \%-100 \%$, as expected, while the propidium iodide ("PI") curve always starts above $0 \%$ and often ends below $100 \%$. The reasons for these differences are explained in the following paragraphs.

\section{0\% Minimum of the Bleomycin Method}

It must be stressed that the $0 \%$ minimum of the bleomycin curves is not a biased artifact introduced by calculation. Without electric pulses, the viability of the cells is not affected even after an 18-h incubation in a culture medium containing 7000-nM bleomycin concentration (1). Likewise, the differences between the cloning efficiency of nonpulsed cells kept in pure suspension and of nonpulsed cells exposed to $5 \mathrm{nM}$ bleomycin for the time of the experiment are statistically insignificant (data not shown). With no exposure to electric pulses, the 5-nM bleomycin method gives $0 \%$ electropermeabilization, as expected.

\section{Above-0\% Minimum of the Propidium Iodide Method}

Cell suspensions necessarily contain a certain fraction of dead cells, essentially due to stress suffered in the handling during the experimental procedure. Since they are not killed by electric pulses, they should be omitted from the evaluation of both cell permeabilization and cell death caused by electric pulses. The bleomycin method excludes these cells automatically because they represent the same fraction in the control as in the assays where pulses are delivered. In the propidium iodide method, those dead cells that have not yet disintegrated fluoresce and appear as electropermeabilized. This inevitably gives an above-zero fraction of electropermeabilized cells in every assay including the control, though electropermeabilization obviously cannot occur if no pulses are delivered.
The propidium iodide curve is often scaled to start at $0 \%$ by subtracting the number of cells detected as permeabilized in the control from those detected as permeabilized in each assay. This approach can nevertheless be questioned, as its results depend on the chosen value of the minimum fluorescence of electropermeabilization (Figure 2).

\section{$100 \%$ Maximum of the Bleomycin Method}

The bleomycin curve always reaches $100 \%$, which implies that all the cells are electropermeabilized at the corresponding pulse amplitude. This is also supported by the fact that the $100 \%$ values are reached at pulse amplitudes significantly below those found to cause $100 \%$ cell killing in the medium with no bleomycin (8). It must be noted that for cell lines with an elevated ability of DNA repair, $5 \mathrm{nM}$ bleomycin might not suffice for the death of all electropermeabilized cells. This would result in a below- $100 \%$ maximum of the bleomycin curve. In these cases, the lowest concentration of bleomycin that yields a $100 \%$ maximum should first be determined, and this concentration should be used for a fully functional method.

\section{Below-100\% Maximum of the Propidium Iodide Method}

Even at very high pulse amplitudes, the bottom right quadrant of the flow cytometer plot often contains a certain number of particles. These are most probably cellular ghosts devoid of DNA (and thus of fluorescence), which can be the remnants of intensively electropermeabilized cells. Unlike the bleomycin curve, the propidium iodide curve often does not reach $100 \%$, which does not reflect the reality.

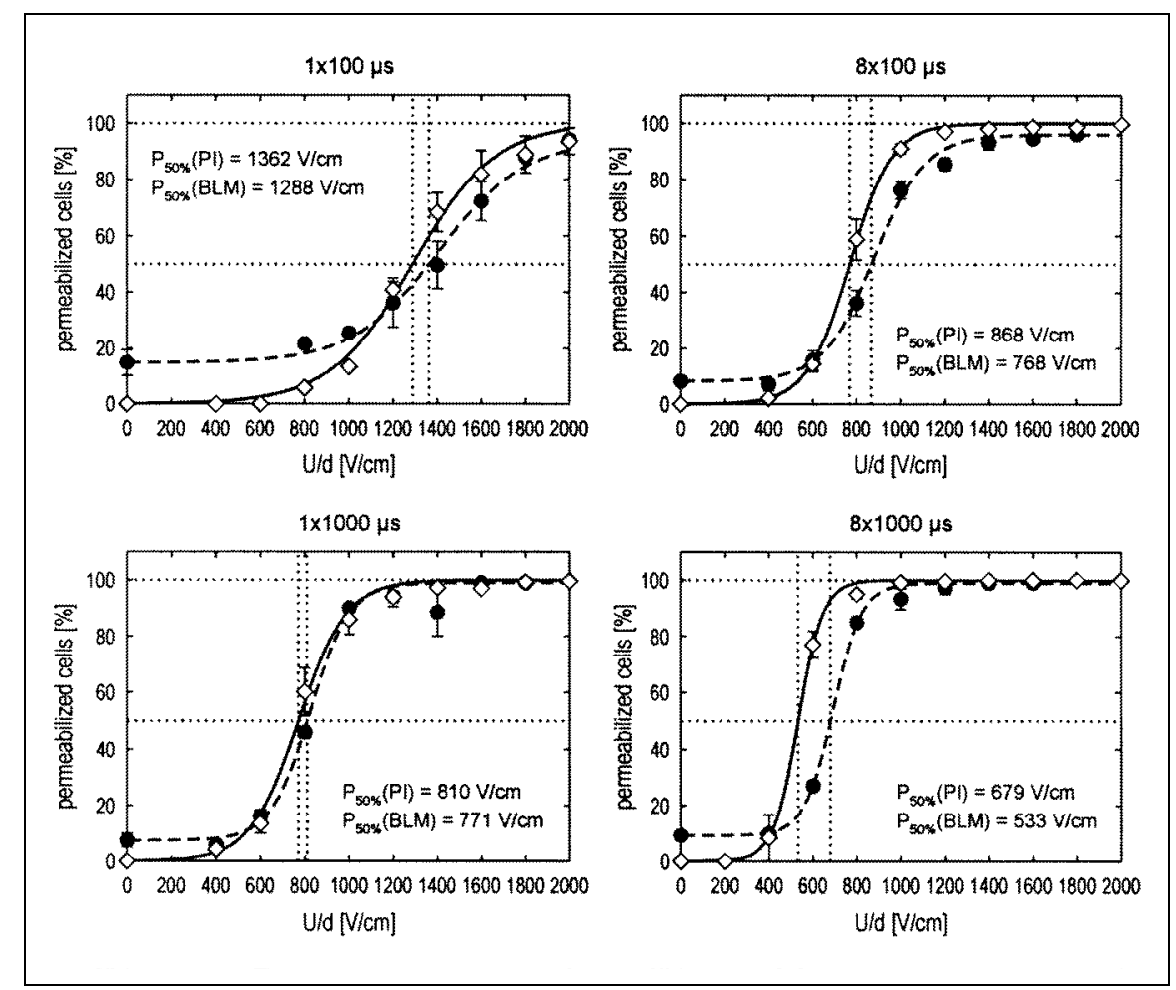

Figure 3. Electropermeabilization as a function of pulse amplitude determined by the bleomycin (BLM) method $(\diamond)$ and by the prodium iodide (PI) method $(\bullet)$. The symbols denote the means, and the error bars are the standard deviations. In each graph, a two-parameter sigmoid curve is fitted to bleomycin data (solid), and a four-parameter sigmoid curve is fitted to propidium iodide data (dashed). For both methods, the sigmoid curves define the corresponding values of $\mathrm{P}_{50 \%}$, which are also displayed. Cell death caused by the pulses alone (i.e., in the absence of bleomycin) only became detectable above $1600 \mathrm{~V} / \mathrm{cm}$ at $1 \times 100-\mu$ s pulse, above $1200 \mathrm{~V} / \mathrm{cm}$ at $8 \times 100-\mu$ s and $1 \times 1000-\mu$ s pulses and above 600 $\mathrm{V} / \mathrm{cm}$ at $8 \times 1000-\mu$ s pulses (data not shown). With the exception of the last, these pulse amplitudes are far above the respective $\mathrm{P}_{50 \%}$ values. 


\section{Other Features of the Bleomycin Method}

The second advantage of the bleomycin method is correct detection of cells disintegrated because of intensive electropermeabilization. The bleomycin method determines cell survival, and the disintegrated cells are evidently included along with the other dead cells. In contrast, the propidium iodide method detects the disintegrated cells in the debris in the top left quadrant. To classify them correctly as permeabilized cells, the number of cells would have to be reconstructed out of the number of debris particles, which is clearly an impossible task.

The third advantage of the bleomycin method is that it needs no arbitrarily chosen limit for the classification of permeabilized cells. With the propidium iodide method, the lower limit of fluorescence intensity must be chosen manually, and an appropriate choice is based entirely on experience. In addition, fluorescence can be affected by the experimental conditions and can also differ between cell lines. The effect of the manual choice of the fluorescence limit is demonstrated in Figure 3, where the $\mathrm{P}_{50 \%}$ values given by the propidium iodide method are invariably higher than the ones provided by the bleomycin method. If a lower value of the limiting fluorescence is chosen, the propidium iodide curves are shifted to the right.

The bleomycin method successfully eliminates these drawbacks of the propidium iodide method, but the latter method still has its merits. First, based on the fluorescence intensity, the propidium iodide method gives an insight into the distribution of the uptake among the cell population (Figure 2). Second, when the results have to be obtained very rapidly, the propidium iodide method only takes hours, while the cloning efficiency determination in the bleomycin method requires five days.

Finally, the fourth advantage of the bleomycin method lies in its affordability. No sophisticated equipment is needed, and a single package of bleomycin, if properly repacked and stored (e.g., frozen), can last for thousands of experiments.

\section{REFERENCES}

1.Akiyama, S., K. Hidaka, S. Komiyama and M. Kuwano. 1979. Control of permeation of bleomycin $\mathrm{A}_{2}$ by polyene antibiotics in cultured Chinese hamster cells. Cancer Res. 39:5150-5154.

2.Arndt-Jovin, D.J. and T.M. Jovin. 1989. Fluorescence labeling and microscopy of DNA. Methods Cell. Biol. 30:417-448.

3.Biedler, J.L. and H. Riehm. 1970. Cellular resistance to actinomycin D in Chinese hamster cells in vitro. Cancer Res. 30:1174-1184.

4.Bobinnec, Y., A. Khodyakov, L.M. Mir, C.L. Rieder, B. Eddé and M. Bornens. 1998. Centriole disassembly in vivo and its effects 
on chromosome structure and function in vertebrate cells. J. Cell Biol. 143:1575-1589.

5.Denizot, F. and R. Lang. 1986. Rapid colorimetric assay for cell growth and survival Modifications to the tetrazolium dye procedure giving improved sensitivity and reliability. J. Immunol. Methods 89:271-277.

6.Dinchuk, J.E., K.A. Kelley and G.N. Callahan. 1992. Flow cytometric analysis of transport activity in lymphocytes electroporated with a fluorescent organic anion dye. J. Immunol. Methods 155:257-265

7.Lukas, J., J. Bartek and M. Strauss. 1994 Efficient transfer of antibodies into mammalian cells by electroporation. J. Immunol. Methods 170:255-259.

8.Mir, L.M., H. Banoun and C. Paoletti. 1988 Introduction of definite amounts of nonpermeant molecules into living cells after electropermeabilization: direct access to the cytosol. Exp. Cell Res. 175:15-25.

9.Mir, L.M., L.F. Glass, G. Serša, J. Teissié, C. Domenge, D. Miklavčič, M.J. Jaroszeski, S Orlowski et al. 1998. Effective treatment of cutaneous and subcutaneous malignant tumors by electrochemotherapy. Br. J. Cancer 77:2336-2342.

10.Mir, L.M., O. Tounekti and S. Orlowski. 1996. Bleomycin: revival of an old drug. Gen. Pharmacol. 27:745-748.

11.Neumann, E., S. Kakorin and K. Toensing. 1999. Fundamentals of electroporative delivery of drugs and genes. Bioelectrochem. Bioenerg. 48:3-16.

12.Rols, M.P. and J. Teissié. 1998. Flow cytometry quantification of electropermeabilization. Methods Mol. Biol. 91:141-147.

13.Roper, P.R. and B. Drewinko. 1976. Comparison of in vitro methods to determine drug-induced cell lethality. Cancer Res. 36:2182-2188.

14.Saunders, J.A., C.H. Lin, B.H. Hou, J. Cheng, N. Tsengwa, J.J. Lin, C.R. Smith, M.S. McIntosh and S. Van Wert. 1995 Rapid optimization of electroporation conditions for plant cells, protoplasts, and pollen. Mol. Biotechnol. 3:181-90.

This work was supported in part by the CNRS, the Institut Gustave-Roussy, the University of Paris XI and by the Ministry of Science and Technology of the Republic of Slovenia. Address correspondence to $\mathrm{Dr}$. Lluis M. Mir, UMR 8532 CNRS, Institut Gustave-Roussy, 39 rue C. Desmoulins, F-94805 Villejuif, France.Internet: luismir@igr.fr

Received 8 November 1999; accepted 24 January 2000.

Tadej Kotnik ${ }^{1,2}$, Alenka

Maček-Lebar ${ }^{1}$, Damijan

Miklavčičr ${ }^{1}$ and Lluis M. Mir ${ }^{2}$

${ }^{1}$ University of Ljubljana

Ljubljana, Slovenia

${ }^{2}$ CNRS/Institut Gustave-Roussy

Villejuif, France

\section{Rapid Determination of Membrane Transport Parameters in Adherent Cells}

BioTechniques 28:926-932 (May 2000)

\section{ABSTRACT}

Reported here is a new method that permits rapid (approximately $5 \mathrm{~s}$ ) determinations of membrane transport phenomena in cells grown in monolayers at the base of 17-mm glass scintillation vials. The method is convenient, cost effective and requires no special apparatus. Initial uptake rates, steady-state and free substrate levels are demonstrated in ZR-75-1 breast cancer and Chinese hamster ovary cell lines using methotrexate, a model agent transported by the reduced folate carrier. The technique should be applicable to the study of the transport properties in a broad range of substrates and cells in monolayer culture.

\section{INTRODUCTION}

Membrane transport of physiological substrates and pharmacological agents plays an important role in determining subsequent anabolic or catabolic events within cells or as a determinant of drug activities. The careful characterization of transport properties (that is, influx kinetics and transmembrane gradients) is difficult to achieve when cells grow only under adherent culture conditions. In particular, it may not be possible to obtain uptake points rapidly enough to capture initial rates and ensure that uptake measurements are not complicated by the metabolism of the transport substrate or its export from cells.

Transport studies for cells in adherent cultures are usually performed in petri dishes or multi-well plates. Some methods have been developed that require special custom-made devices $(7,16)$. Besides being costly and complex, these techniques can be subject to fluctuations in incubation temperatures within the laboratory or they may require an ambient temperature of $37^{\circ} \mathrm{C}$. Hence, transport studies for adherent cells are often limited to single uptake points or long intervals with fewer time points $(12,14,15)$. This report describes a rapid, cost-effective method that simplifies the study of transport in cells in adherent culture, permits the study of early uptake points and allows a level of accuracy and precision that is necessary for the quantitative analysis of transport parameters under physiological growth conditions.

\section{MATERIALS AND METHODS}

\section{Materials}

Materials were obtained as follows: Iscove's Modified Dulbecco's Medium (IMDM) (HyClone, Logan, UT, USA); fetal bovine serum (Gemini BioProducts, Calabasas, CA, USA); trypsin (Life Technologies, Rockville, MD, USA); hygromycin B (CalbiochemNovabiochem, San Diego, CA, USA); methotrexate (MTX) (Lederle, Carolina, PR); $\quad\left[3^{\prime}, 5^{\prime}, 7^{\prime}-3 \mathrm{H}\right]$ methotrexate (Amersham Pharmacia Biotech, Piscataway, NJ, USA); low-background 17mm scintillation glass vials (Research Products International, Mt. Prospect, IL, and Fisher Scientific Pittsburg, PA,USA); 8-mL culture vials (Lawson Mardon Wheaton, Millville, NJ, USA); and BCA protein assay reagent kit (Pierce Chemical, Rockford, IL, USA).

\section{Cell Lines and Growth Conditions}

Wild-type ZR-75-1 (5) and the human RFC1-transfected MTX ${ }^{\mathrm{R}}$ ZR75/RFC1 human breast cancer cell lines (12) were maintained in $25-\mathrm{cm}^{2}$ culture flasks in IMDM supplemented with 5\% fetal bovine serum, $2 \mathrm{mM}$ L-glutamine, $100 \mathrm{U} / \mathrm{mL}$ penicillin and $100 \mu \mathrm{g} / \mathrm{mL}$ streptomycin. PYR $100 / \mathrm{RFC} 1$, a $\mathrm{CHO}$ cell line transfected with murine RFC1, was maintained in RPMI 1640 medium supplemented with $5 \%$ fetal bovine serum (1). Hygromycin B $(150 \mu \mathrm{g} / \mathrm{mL})$ was added to the growth medium of the transfected cells.

\section{Purification of Methotrexate}

Tritiated and nonlabeled MTX were purified by reverse phase HPLC as described previously (11). 